\title{
Rapidity dependence of entropy production in proton- and nucleus-induced reactions on heavy nuclei
}

\author{
B. V. Jacak, H. Stöcker, and G. D. Westfall \\ National Superconducting Cyclotron Laboratory, Michigan State University, East Lansing, Michigan 48824
}

(Received 13 December 1983)

\begin{abstract}
The entropy of hot nuclear systems is deduced from the mass distribution of fragments emitted from high energy proton- and nucleus-induced reactions via a quantum statistical model. It is found that the entropy per baryon, $S / A$, of intermediate rapidity ("participant") fragments is higher than the entropy of target rapidity ("spectator") fragments. The spectator fragments exhibit $S / A$ values of $\approx 1.8$ independent of the projectile energy from $30 \mathrm{MeV} /$ nucleon up to $350 \mathrm{GeV}$. This value of the entropy coincides with the entropy at which nuclear matter becomes unbound.
\end{abstract}

The study of the entropy produced in proton- and heavy ion-induced reactions on heavy nuclei can provide a method of studying the collective behavior of nuclear matter. ${ }^{1-4}$ Hydrodynamical $^{5,6}$ and intranuclear cascade calculations $^{7}$ have demonstrated that the entropy per baryon, $S / A$, created in high energy nucleus-nucleus collisions remains constant during the expansion of the system. Information concerning the entropy produced in these reactions can be obtained by comparing the fragment production cross sections with a quantum statistical model. Thus fragments observed experimentally can carry information concerning not only the late stages of the reaction but also the hot, compressed stage of the reaction.

In high energy proton-nucleus interactions, the target is excited and decays by emitting nucleons and nuclear fragments as well as fissioning and emitting $\gamma$ rays. In central collisions of high energy heavy ions, a compressed and highly excited interaction region ${ }^{4,8,9}$ is formed which subsequently expands and cools. ${ }^{3}$ The system expands until the constituents are no longer interacting. At this time the momentum distributions and particle concentrations are frozen out. Particles emitted from this system appear predominantly at rapidities intermediate between those of the projectile and target, while fragments from protonnucleus and peripheral nucleus-nucleus reactions are emitted at rapidities close to the projectile and target rapidities.

The entropy created in high energy proton- and nucleus-induced reactions can be extracted from the resulting fragment production cross sections using the quantum statistical model as proposed in Ref. 3. This method was used in Ref. 4 to determine the entropy via the intermediate rapidity fragments $1 \leq A \leq 14$ emitted from high energy nucleus-nucleus reactions. The quantum statistical model assumes a system of $N$ neutrons and $Z$ protons in thermal and chemical equilibrium at a given entropy per baryon $S / A$ and nuclear density, $\rho$, which then specifies the temperature $\tau$. Included in the calculation are nucleons, pions, delta resonances, stable and $\gamma$ unstable nuclei up to $A=20$, and the known particle unstable nuclear states up to $A=10$. The predictions of this model become less reliable above $A=10$, thus compar- isons with data are done only up to $A=14$. The $N$ and $Z$ of the initial system are chosen to be those of the target nucleus for proton-induced and peripheral nucleusinduced reactions. For nucleus-nucleus reactions the overlapping volume of the two nuclei at the impact parameter with the most weight is used. Interactions are taken into account via the excluded volume of the fragments. The $S / A$ for a given beam, target, and incident energy is extracted using a least squares fit to the observed fragment distributions. The fragment distributions calculated for a given $S / A$ are insensitive to the assumed breakup density for $0.3 \rho_{0} \leq \rho \leq 0.7 \rho_{0} \cdot{ }^{3,4}$

A large body of data exists concerning high energy proton induced reactions on heavy nuclei. We concentrate here on fragmentation or spallation of target nuclei leading to the emission of fragments with $1 \leq A \leq 40 .{ }^{10-12}$ These data are complemented with measurements of target rapidity fragments from high energy nucleus-induced reactions., ${ }^{913-15}$ Radiochemical measurements will not be discussed here because they generally report data for fragments with $A \geq 40$. In Fig. 1 relative production cross sections are shown for target rapidity from the reactions $400 \mathrm{MeV} /$ nucleon $\mathrm{Ne}+\mathrm{U},{ }^{9} 2.1 \mathrm{GeV} /$ nucleon $\mathrm{Ne}+\mathrm{Au},{ }^{14}$ $480 \mathrm{MeV} p+\mathrm{Ag}^{12}$ and $80-350 \mathrm{GeV} \mathrm{p}+\mathrm{Xe}^{10}$ The solid lines in Fig. 1 represent the quantum statistical calculation described above carried out at a density of $\rho=0.3 \rho_{0}$. Although data were measured up to $A=30$ for the $\mathrm{p}+\mathrm{Xe}$ case, only the cross sections that can be compared with the present model are shown. The fits agree well with the observed fragment yields for all four cases, except for $Z=2$ fragments from $480 \mathrm{MeV} \mathrm{p}+\mathrm{Ag}$. The entropies obtained from the fits to these fragment distributions as well as those extracted from the reactions of $30 \mathrm{MeV} /$ nucleon $\mathrm{C}+\mathrm{Au},^{13} 55-110 \mathrm{MeV} /$ nucleon $\mathrm{C}+\mathrm{Ag}^{15}{ }^{15} 250 \mathrm{MeV} /$ nucleon $^{\mathrm{Ne}}+\mathrm{Au},{ }^{14} 2100$ $\mathrm{MeV} /$ nucleon $\mathrm{Ne}+\mathrm{U},{ }^{9}$ and $4.9 \mathrm{GeV} \mathrm{p}+\mathrm{Ag}$ and $\mathrm{U}$ (Ref. 11) are shown in Fig. 2(a) as a function of the projectile kinetic energy/nucleon. The depicted errors reflect the errors from the fit as well as known systematic errors in the data. The fits generally encompass fragments with $3 \leq Z \leq 10$ and appear to be independent of both projectile-type and energy. The average value for $S / A$ in these cases is $1.84 \pm 0.16$. Not shown in this figure is $S / A$ 

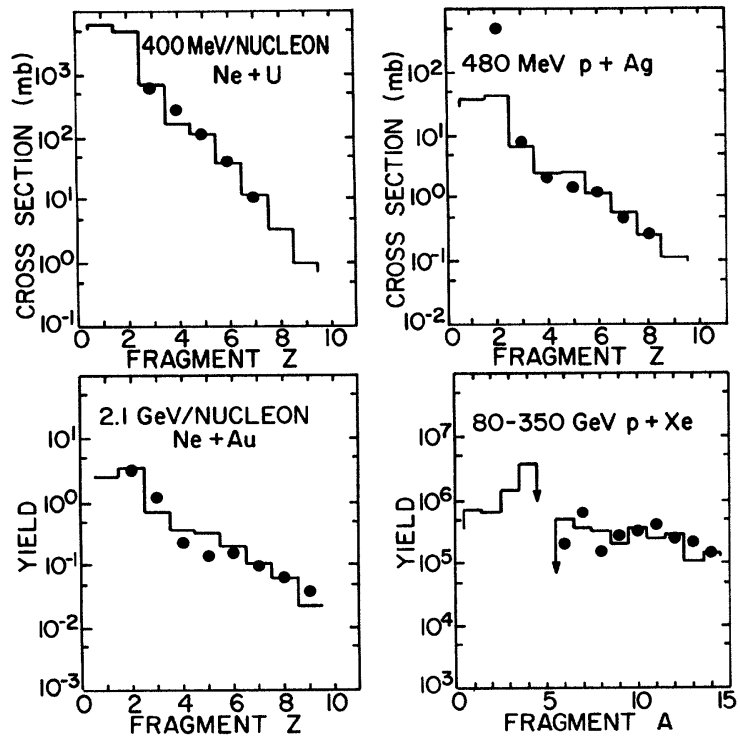

FIG. 1. Target rapidity fragments from the reactions of (a) $400 \mathrm{MeV} /$ nucleon $\mathrm{Ne}+\mathrm{U}$ (Ref. 9), (b) $480 \mathrm{MeV} \mathrm{p}+\mathrm{Ag}$ (Ref. 12), (c) $2.1 \mathrm{GeV} /$ nucleon $\mathrm{Ne}+\mathrm{Au}$ (Ref. 14), and (d) $80-350$ GeV p + Xe (Ref. 10). The histograms represent fits using a quantum statistical calculation as described in the text.

extracted from $80-350 \mathrm{GeV} \mathrm{p}+\mathrm{Xe}$ (Ref. 10) which is $1.46 \pm 0.67$. This constant value of 1.84 for the extracted $S / A$ coincides with the entropy necessary to excite the target nuclei to their binding energy.

Shown in Fig. 2(b) are the extracted entropies for intermediate rapidity fragments $(1 \leq A \leq 14)$ from the reaction of $\mathrm{Ar}+\mathrm{Au}$ at 42,92 , and $137 \mathrm{MeV} /$ nucleon. These values are higher than those extracted from target fragments and increase somewhat with bombarding energy. ${ }^{4}$ No data exist for intermediate rapidity fragments with $A \geq 6$ at energies above $137 \mathrm{MeV} /$ nucleon. The data of Ref. 9 do not extend to intermediate rapidities for fragments heavier than ${ }^{4} \mathrm{He}$.

One may treat these fragments by assuming production in a fireball created by the overlapping volume of the projectile nucleus with the target nucleus. The excitation of the fireball can be calculated taking into account the slowing from Coulomb repulsion between the two nuclei and the resulting entropy can be calculated using the Fermi gas model. These calculations are shown in Fig. 2(b) as solid lines for three densities, $\rho=1.0 \rho_{0}, 2.0 \rho_{0}$, and $3.0 \rho_{0}$. The maximum density of the fireball system will increase with beam energy and a more sophisticated model such as hydrodynamics would incorporate this effect.

The extracted entropies from intermediate rapidity fragments with $1 \leq A \leq 3$ and $1 \leq A \leq 4$ from high energy nucleus-nucleus reactions are also shown in Fig. 2(b). Typical fits to intermediate rapidity light particles only are shown in Fig. 3. In Fig. 3(a) light fragments with $1 \leq A \leq 4$ from the reaction of $137 \mathrm{MeV} /$ nucleon $\mathrm{Ar}+\mathrm{Au}$ (Ref. 4) are fitted, while fragments with $1 \leq A \leq 4$ and $1 \leq A \leq 3$ are fitted in Figs. 3(b) and 3(c), respectively, for the sytsem $393 \mathrm{MeV} /$ nucleon $\mathrm{Ne}+\mathrm{U}^{17}$ Additional systems reported in Fig. 2(b) are $35 \mathrm{MeV} /$ nucleon $\mathrm{C}+\mathrm{Au},{ }^{16}$ 42, 92, and $137 \mathrm{MeV} /$ nucleon $\mathrm{Ar}+\mathrm{Au}^{4}{ }^{4} 100$ and 156

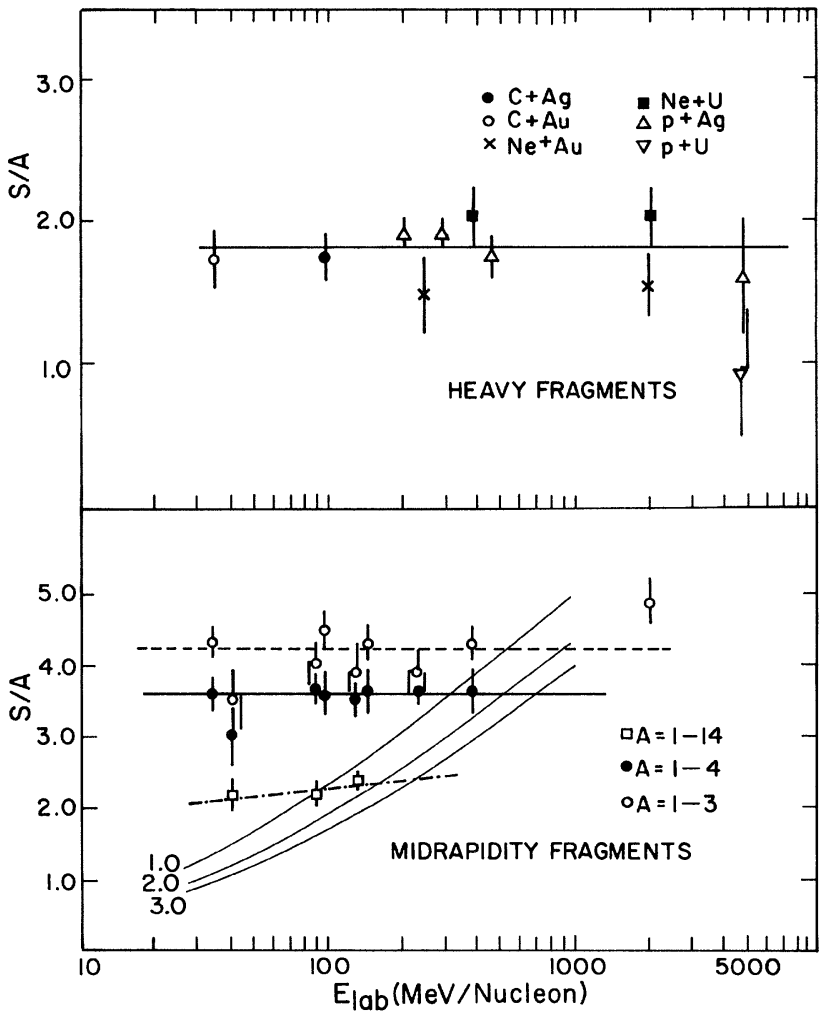

FIG. 2. Extracted entropy per baryon $(S / A)$ using a quantum statistical model as described in the text. (a) Target rapidity fragments from the reactions $480 \mathrm{MeV} \mathrm{p}+\mathrm{Ag}$ (Ref. 12), 2.1 $\mathrm{GeV} /$ nucleon $\mathrm{Ne}+\mathrm{Au}$ (Ref. 14), $30 \mathrm{MeV} /$ nucleon $\mathrm{C}+\mathrm{Au}$ (Ref. 13), 55-110 MeV/nucleon C + Ag (Ref. 15), 250 and $2100 \mathrm{MeV} /$ nucleon $\mathrm{Ne}+\mathrm{Au}$ (Ref. 14), 400 and 2100 $\mathrm{MeV} /$ nucleon $\mathrm{Ne}+\mathrm{U}$ (Ref. 9), and $4.9 \mathrm{GeV} \mathrm{p}+\mathrm{Ag}$ and $\mathrm{U}$ (Ref. 11); (b) midrapidity fragments from the reactions 35 $\mathrm{MeV} /$ nucleon $\mathrm{C}+\mathrm{Au}$ (Ref. 16), 42, 92, and $137 \mathrm{MeV} /$ nucleon $\mathrm{Ar}+\mathrm{Au}$ (Ref. 4), 100 and $156 \mathrm{MeV} /$ nucleon $\mathrm{Ne}+\mathrm{Au}$ (Ref. 8), 241 and $393 \mathrm{MeV} /$ nucleon $\mathrm{Ne}+\mathrm{U}$ (Ref. 17), and 2.1 $\mathrm{GeV} /$ nucleon $\mathrm{Ne}+\mathrm{Pb}$ (Ref. 18). The dashed and solid lines represent the weighted average for fragments with $1 \leq A \leq 3$ and $1 \leq A \leq 4$, respectively. The dot-dash line represents a power law fit for fragments with $1 \leq A \leq 14$. The three grouped solid lines represent the entropy calculated using fireball geometry and a Fermi gas model at three different densities, $\rho=1.0 \rho_{0}$, 2. $0 \rho_{0}$, and $3.0 \rho_{0}$.

$\mathrm{MeV} /$ nucleon $\mathrm{Ne}+\mathrm{Au},{ }^{8} 241$ and $393 \mathrm{MeV} /$ nucleon $\mathrm{Ne}+\mathrm{U},{ }^{17}$ and $2.1 \mathrm{GeV} /$ nucleon $\mathrm{Ne}+\mathrm{Pb} .{ }^{18}$ The average value of $S / A$ for fragments with $1 \leq A \leq 3$ and $1 \leq A \leq 4$ are $4.24 \pm 0.32$ and $3.60 \pm 0.12$, respectively, independent of the incident energy and projectile nucleus. These values can be compared to the entropies extracted from proton and deuteron cross sections using a model in which the system is assumed to be a dilute gas of nucleons and deuterons ${ }^{1}$ where an $S / A$ of $=4.7$ was deduced.

The difference between the entropy extracted using the same quantum statistical model compared to light particle cross sections and cross sections for fragments with $1 \leq A \leq 4$ appears to be a paradox because these fragments seem to have common origins. The apparent temperatures of these fragments are similar to each other at a 

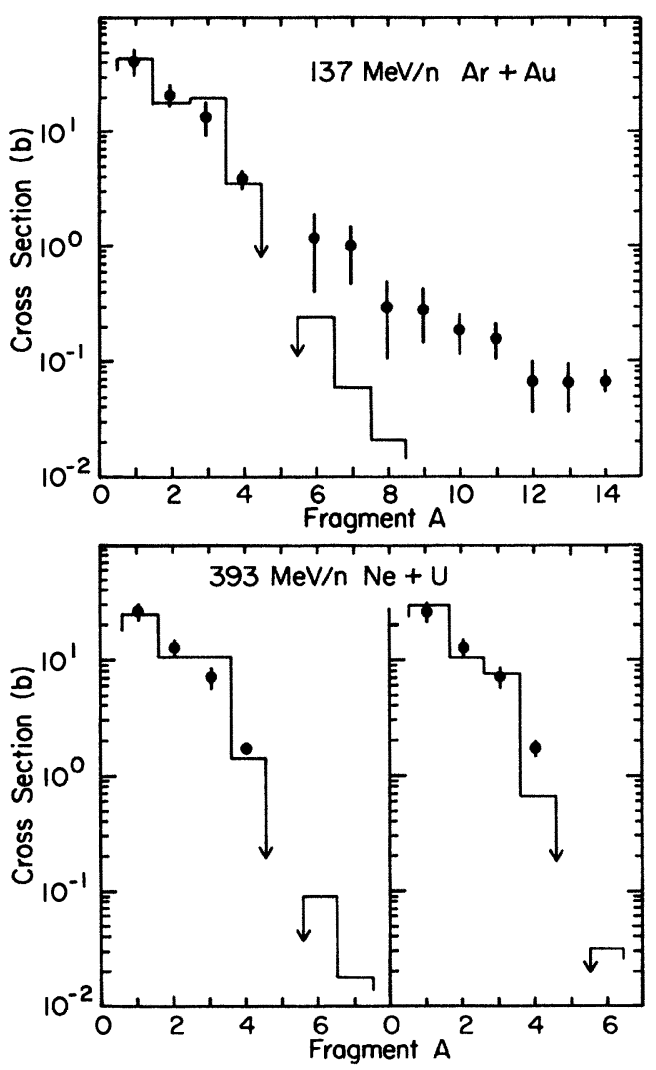

FIG. 3. Intermediate rapidity fragments from 137 $\mathrm{MeV} /$ nucleon $\mathrm{Ar}+\mathrm{Au}$ (Ref. 4) and $393 \mathrm{MeV} /$ nucleon $\mathrm{Ne}+\mathrm{U}$ (Ref. 9). The solid lines represent fits using a quantum statistical model as described in the text where fragments with $1 \leq A \leq 4$ are fitted in (a) and (b) while in (c) only fragments with $1 \leq A \leq 3$ are fitted.

given bombarding energy, while the extracted source velocities vary from 0.5 times the projectile velocity for $A \leq 3$ to 0.3 times the projectile velocity for the heavier fragments. However, the present light particle inclusive data are dominated by more peripheral collisions where the small number of nucleons contained in the interaction volume between the two nuclei exclude the formation of heavier emitted fragments. The macrocanonical approach inherent in the quantum statistical model requires many particles in the system and is inapplicable for peripheral collisions. In contrast, the thermodynamic limit is approached for near-central collisions ${ }^{2}$ which is where medium mass fragments are produced. ${ }^{14}$ It is clear that to extract the entropy from high energy nucleus-nucleus reactions it is essential to treat complex fragments as well as light particles.

The distributions of fragment masses from high energy proton- and nucleus-induced reactions have been previously interpreted in terms of a power law dependence on the mass number of the emitted fragment ${ }^{10}$ which would be indicative of a liquid-vapor phase transition $3,10,13,14,19-21$. It is clear from the present work and other work ${ }^{22,23}$ that the mass yields can be described without the need to invoke a liquid-vapor phase transition.

In summary, the entropy produced in high energy proton- and nucleus-induced reactions on heavy nuclei is extracted using a quantum statistical model for the emission of complex fragments. The $S / A$ extracted from targetlike fragments is $1.84 \pm 0.16$ independent of beam energy, projectile, and target. For intermediate rapidity complex fragments from nucleus-nucleus reactions, $S / A$ tends to increase with bombarding energy from 2.2 at 42 $\mathrm{MeV} /$ nucleon to 2.4 at $137 \mathrm{MeV} /$ nucleon. However, the lack of intermediate rapidity complex fragment data at higher energies hampers a definite conclusion concerning the energy dependence of $S / A$ from these systems. Entropies extracted for intermediate rapidity light particles appear to be independent of beam energy, projectile, and target mass and are in the range of $S / A=3-5$. This effect could be due to the fact that inclusive data were used to extract the entropy. These cross sections are biased toward smaller reaction volumes with a correspondingly smaller number of participants. ${ }^{2}$ Only by measuring particles from impact parameter selected events can the ambiguity between the light and heavy fragments be resolved.

The authors wish to acknowledge stimulating discussions with D. H. Boal, J. I. Kapusta, H. Kruse, and D. K. Scott. This work was supported by the National Science Foundation, Grant No. PHY 83122455.
1P. Siemens and J. I. Kapusta, Phys. Rev. Lett. 43, 1486 (1979).

${ }^{2}$ H. H. Gutbrod, H. Löhner, A. M. Poskanzer, T. Renner, H. Riedesel, H. G. Ritter, A. Warwick, F. Weik, and H. Wieman, Phys. Lett. 127B, 317 (1983).

${ }^{3}$ H. Stöcker, G. Buchwald, G. Graebner, P. Subramanian, J. A. Maruhn, W. Greiner, B. V. Jacak, and G. D. Westfall, Nucl. Phys. A400, 63 (1983).

${ }^{4}$ B. V. Jacak, G. D. Westfall, C. K. Gelbke, L. H. Harwood, W. G. Lynch, D. K. Scott, H. Stöcker, M. B. Tsang, and T. J. M. Symons, Phys. Rev. Lett. 51, 1846 (1983).

${ }^{5}$ H. Stöcker, J. Hofmann, J. A. Maruhn, and W. Greiner, Prog. Part. Nucl. Phys. 4, 133 (1980).

6J. I. Kapusta and D. Strottman, Phys. Rev. C 23, 1282 (1981).

${ }^{7}$ G. Bertsch and J. Cugnon, Phys. Rev. C 24, 2514 (1981).

${ }^{8}$ G. D. Westfall, B. V. Jacak, N. Anantaraman, M. W. Curtin, G. M. Crawley, C. K. Gelbke, B. Hasselquist, W. G. Lynch, D. K. Scott, M. B. Tsang, M. J. Murphy, T. J. M. Symons, R.
Legrain, and T. J. Majors, Phys. Lett. 116B, 118 (1982).

9J. Gosset, H. H. Gutbrod, W. G. Meyer, A. M. Poskanzer, A. Sandoval, R. Stock, and G. D. Westfall, Phys. Rev. C 16, 629 (1977).

10J. E. Finn, S. Agarwal, A. Bujak, J. Chuang, L. J. Gutay, A. S. Hirsch, R. W. Minich, N. T. Porile, R. P. Scharenberg, B. C. Stringfellow, and F. Turkot, Phys. Rev. Lett. 49, 1321 (1982).

${ }^{11}$ G. D. Westfall, R. G. Sextro, A. M. Poskanzer, A. M. Zebelman, G. W. Butler, and E. K. Hyde, Phys. Rev. C 17, 1368 (1978), and references therein.

${ }^{12}$ Ray E. L. Green and Ralph G. Korteling, Phys. Rev. C 22, 1594 (1980).

${ }^{13}$ C. B. Chitwood, D. J. Fields, C. K. Gelbke, W. G. Lynch, A. D. Panagiotou, M. B. Tsang, H. Utsunomiya, and W. A. Friedman, Phys. Lett. 131B, 289 (1983).

${ }^{14}$ A. I. Warwick, H. H. Wieman, H. H. Gutbrod, M. R. Maier, 
J. Peter, H. G. Ritter, H. Stelzer, F. Weik, M. Freedman, D. J. Henderson, S. B. Kaufman, E. P. Steinberg, and B. D. Wilkins, Phys. Rev. C 27, 1083 (1983).

${ }^{15}$ B. Jakobsson, G. Jönsson, B. Lindkvist, and A. Oskarsson, Z. Phys. A 307, 293 (1982).

${ }^{16}$ G. D. Westfall, Z. M. Koenig, B. V. Jacak, L. H. Harwood, G. M. Crawley, M. W. Curtin, C. K. Gelbke, B. Hasselquist, W. G. Lynch, A. D. Panagiotou, D. K. Scott, H. Stöcker, and M. B. Tsang, Phys. Rev. C 29, 861 (1984).

${ }^{17}$ A. Sandoval, H. H. Gutbrod, W. G. Meyer, R. Stock, Ch. Lukner, A. M. Poskanzer, J. Gosset, J.-C. Jourdain, C. H. King, Nguyen Van Sen, G. D. Westfall, and K. L. Wolf,
Phys. Rev. C 21, 1321 (1980).

${ }^{18}$ S. Nagamiya, M.-C. Lemaire, E. Moeller, S. Schnetzer, G. Shapiro, H. Steiner, and I. Tanihata, Phys. Rev. C 24, 971 (1981).

${ }^{19}$ P. J. Siemens, Nature $\underline{305}, 410$ (1983).

${ }^{20}$ A. D. Panagiotou, M. W. Curtin, H. Toki, D. K. Scott, and P. J. Siemens, Phys. Rev. Lett. 52, 496 (1984).

${ }^{21}$ A. L. Goodman, J. I. Kapusta, and A. Z. Mekjian, Lawrence Berkeley Laboratory Report LBL-16471, 1983.

${ }^{22}$ W. A. Friedman and W. G. Lynch, Phys. Rev. C 28, 950 (1983).

${ }^{23}$ D. H. Boal (unpublished). 